\title{
A Numerical Study on Passive Control of Shock Wave/Turbulent Boundary Layer in a Supersonic Compressor Cascade
}

\author{
J. I. Seo, S. D. Kim, and D. J. Song \\ School of Mechanical Engineering, Yeungnam University, Gyongsan, Korea
}

\begin{abstract}
A finite-difference method based on upwind flux difference splitting has been used to predict the performance of supersonic axial compressor cascades and to examine the complex physical flow phenomena. The prediction with Abe, Kondoh, and Nagano's two-equation turbulence model showed good agreement with the experimental results near the zone of shock-wave/turbulent boundary-layer interaction at different exit-inlet pressure ratios. The increase in exit-inlet pressure ratio caused slight reduction in shock loss but an increase in viscous loss. The passive control of shockwave interaction with turbulent boundary layer by a cavity reduced total pressure losses and strength of shock waves. A cavity under the shock foot point at suction surface of the blades was used as a passive control. The effect of cavity length and depth on total pressure loss was studied. The proper size of cavities could reduce total pressure losses even though it increased viscous losses through the formation of shock-wave/turbulent boundary-layer zone.
\end{abstract}

Keywords Shock-wave/turbulent boundary layer interaction; Transonic/supersonic compressor cascade; Cavity; CSCM upwind flux difference splitting algorithm; Abe et al.'s turbulence model; Passive control

The performance prediction of transonic/supersonic compressors and cascades has been a very important research area due to the trends toward high pressure ratio and compact aircraft engines with a reduced number of stages and increased mass flux.

Received 12 March 2001; accepted 14 May 2001.

This paper was partially supported by 1999 Research Fund and Yeungnam University BK21(Brain Korea 21) project.

Address correspondence to Dong Joo Song, Ph.D., School of Mechanical Engineering, Yeungnam University, Gyongsan 712-160, Korea.
It is interesting to investigate the effectiveness of passive control of the shock-wave/turbulent boundary-layer interaction in a transonic/supersonic cascade because its aerodynamic performance is essentially dependent on both the total pressure losses of the shock wave and the momentum losses. The main idea is the use of the static pressure jump over the shock wave to bypass a small secondary flow through a cavity under the shock wave foot point. The passive cavity concept was experimentally demonstrated by Bahi, Ross, and Nagamatsu (1983) for reducing drag on a supercritical airfoil. Nagamatsu, Orozco, and Ling (1984) found that the drag over a supercritical airfoil could be reduced by implementing a passive porous cavity in the region of the shock wave. Their encouraging results have led to many experimental and analytic investigations that have been summarized fairly recently by Raghunathan (1989). McCormick (1993) showed that the passive cavity substantially reduced the total pressure loss through the shock system by causing a more isentropic compression-however, the boundary layer viscous losses downstream of the shock wave were significantly increased. $\mathrm{Nu}$ merical and experimental results of passive control by a cavity covered with a porous plate on the compressor cascades yielded a reduction of the total pressure loss and a slightly improved isentropic efficiency as shown by Schnerr and Dohrmann (1993). The total pressure loss $\omega=\left(p_{t 1}-p_{t 2}\right) /\left(p_{t 1}-p_{1}\right)$ decreased by a few percent due to the porous cavity. Though we are not using the same porous cavity model of Schnerr and Dohrmann, we may obtain qualitatively similar results with a pure cavity of similar size.

The low Reynolds number $k-\varepsilon$ formulation of two-equation turbulence models was quite widely adopted in practical engineering problems due to its robustness. A $k-\varepsilon$ turbulence model was proposed by Abe, Kondoh, and Nagano (1994), whose main improvement was the employment of the Kolmogorov velocity scale $(\nu \varepsilon)^{1 / 4}$ instead of the conventional friction velocity $u_{\tau}$. Their model performed well in solving a diffuser flow with a strong adverse pressure gradient, flow separation, and reattachment of a backward-facing step. This model appreciates 
the motion of a small-scale eddy as dissipating kinetic energy to heat by the action of molecular viscosity that is independent of the relatively slow motion of mean flow and large eddies, which have been mainly considered in developing a typical turbulence closure. Kim and Song (1997) showed that the $k-\varepsilon$ model of Abe, Kondoh, and Nagano performed better in predicting the complex shock-wave/turbulent boundary-layer interaction phenomena in transonic/supersonic flows over axisymmetric bump and compression ramp than other two-equation models did.

Classical upwind schemes can roughly be divided into two categories: flux vector splitting schemes (Steger and Warming, 1981; Van Leer, 1982) and flux difference splitting schemes (Godunov, 1959; Roe, 1981; Osher, 1984). Among the upwind flux difference splitting Navier-Stokes method, CSCM formulation (Lombard et al., 1983) shows good performance in solving 2-/3-D complex high speed flow. It has the merits of upwind scheme, easiness of applying characteristic boundary conditions, and being a fast solver. For ARL-SL19 supersonic compressor cascade flow, Kim et al. (1995) have studied the effects of passive control of shock-wave/laminar boundary-layer interaction in the cascade flowfield. In this study, the CSCM upwind flux difference splitting algorithm with two-equation turbulence model is used to study the influence of passive control by a cavity on the supersonic cascade flow.

We will use Abe, Kondoh, and Nagano's turbulence model and various cavities to control shock-wave/turbulent boundarylayer interaction.

\section{NUMERICAL METHODS}

A finite difference formulation of the compressible NavierStokes equations with fully coupled turbulence model equation (Abe, Kondoh, and Nagano, 1994) can be expressed as

$$
\frac{\partial q}{\partial t}+\frac{\partial F}{\partial x}+\frac{\partial G}{\partial y}=\frac{\partial F_{v}}{\partial x}+\frac{\partial G_{v}}{\partial y}+S
$$

where, conservative dependent variables $q$, inviscid fluxes $F, G$, viscous fluxes $F_{v}, G_{v}$

$$
\begin{gathered}
q=\left(\begin{array}{c}
\rho \\
\rho u \\
\rho v \\
\varepsilon_{t} \\
\rho k \\
\rho \varepsilon
\end{array}\right), \quad F=\left(\begin{array}{c}
\rho u \\
\rho u^{2}+p \\
\rho u v \\
u\left(\varepsilon_{t}+p\right) \\
\rho u k \\
\rho u \varepsilon
\end{array}\right), \quad G=\left(\begin{array}{c}
\rho v \\
\rho u v \\
\rho v^{2}+p \\
v\left(\varepsilon_{t}+p\right) \\
\rho v k \\
\rho v \varepsilon
\end{array}\right) \\
F_{v}=\left(\begin{array}{c} 
\\
0 \\
\tau_{x x} \\
\tau_{y x} \\
u \tau_{x x}+v \tau_{y x}-q_{x} \\
\left(\mu+\mu_{t} / \sigma_{k}\right) k_{x} \\
\left(\mu+\mu_{\varepsilon} / \sigma_{\varepsilon}\right) \varepsilon_{x}
\end{array}\right), \quad G_{v}=\left(\begin{array}{c}
\tau_{y y} \\
u \tau_{x y}+v \tau_{y y}-q_{y} \\
\left(\mu+\mu_{t} / \sigma_{k}\right) k_{y} \\
\left(\mu+\mu_{\varepsilon} / \sigma_{\varepsilon}\right) \varepsilon_{y}
\end{array}\right)
\end{gathered}
$$

and source term $S$

$$
S=\left(\begin{array}{c}
0 \\
0 \\
0 \\
0 \\
P_{k}-\rho \varepsilon \\
c_{1} f_{1} \frac{\varepsilon}{k} P_{k}-c_{2} f_{2} \frac{\rho \varepsilon^{2}}{k}
\end{array}\right)
$$

where $\sigma_{k}=1.6, \sigma_{\phi}=1.6, c_{1}=1.9, c_{2}=1.9$. The damping functions are

$$
\begin{aligned}
& f_{\mu}=\left[1-\exp \left(\frac{-y^{*}}{14}\right)\right]^{2} \times\left[1+\left(\frac{5}{R_{t}^{3 / 4}}\right) \exp \left[-\left(\frac{R_{t}}{200}\right)^{2}\right]\right] \\
& f_{1}=1, f_{2}=\left[1-\exp \left(\frac{-y^{*}}{3.1}\right)\right]^{2} \times\left[1-0.3 \exp \left[-\left(\frac{R_{t}}{6.5}\right)^{2}\right]\right]
\end{aligned}
$$

Turbulence kinetic energy production and the eddy viscosity are given by

$$
\begin{aligned}
& P_{k}=\mu_{t}\left[\frac{1}{2}\left(\frac{\partial u_{i}}{\partial x_{j}}+\frac{\partial u_{j}}{\partial x_{i}}\right)^{2}-\frac{2}{3}\left(\frac{\partial u_{m}}{\partial x_{m}}\right)^{2}\right]-\frac{2}{3} \rho k \frac{\partial u_{m}}{\partial x_{m}} \\
& \mu_{t}=c_{\mu} f_{v} \rho \frac{k^{2}}{\varepsilon}
\end{aligned}
$$

The CSCM upwind flux difference splitting method utilizes the properties of similarity transformation based on conservative, primitive, and characteristic variables:

$$
\begin{aligned}
\Delta F & =A \Delta q=\overline{M T} \Lambda \overline{T^{-1} M^{-1}} \Delta q \\
& =\overline{M T} \Lambda \overline{T^{-1}} \Delta \tilde{q}=\bar{M} \overline{A^{\prime}} \Delta \tilde{q} \\
& =\overline{M T} \Lambda \Delta \tilde{\tilde{q}}
\end{aligned}
$$

where '-' is the simple average of the space. $\tilde{q}$ and $\tilde{\tilde{q}}$ are primitive variables and characteristic variables, respectively. $\Lambda$ is a diagonal matrix whose diagonal elements correspond to the eigenvalues $(u, u, u+c, u-c, u, u)$, and variables $q, \tilde{q}$ and $\tilde{\tilde{q}}$ are related as follows:

$$
\Delta \tilde{q}=\overline{M^{-1}} \Delta q, \quad \Delta \tilde{\tilde{q}}=\overline{T^{-1}} \Delta \tilde{q}
$$

$\overline{M^{-1}}$ matrix transforms conservative variables into primitive variables. $\overline{T^{-1}}$ is a matrix which transforms primitive variables into characteristic variables and it is chosen somewhat arbitrary as to scaling that leads to logarithmic difference approximations for density, pressure, and Mach number.

The characteristic variables can be obtained from the primitive variables through the following relation

$$
\overline{T^{-1}}\left(\overline{A^{\prime}} \Delta \tilde{q}\right)=\overline{T^{-1}}\left(\bar{T} \Lambda \overline{T^{-1}}\right) \Delta \tilde{q}=\Lambda \Delta \tilde{\tilde{q}}
$$

The inviscid flux $\Delta F$ can be divided into $\Delta F^{+}$and $\Delta F^{-}$using diagonal truth function matrix $D^{ \pm}$and Equation (3) can be 
written as

$$
\Delta F=\overline{M T}\left(D^{+}+D^{-}\right) \overline{T^{-1}} \bar{A}^{\prime} \Delta \tilde{q}=\Delta F^{+}+\Delta F^{-}
$$

where $D^{+}=\frac{1}{2}\left[\frac{\Lambda}{|\Lambda|}+1\right], D^{-}=\frac{1}{2}\left[1-\frac{\Lambda}{|\Lambda|}\right]$. Using the relation $\bar{A}^{\prime} \Delta \tilde{q}=\widetilde{M^{-1}} \Delta q$, the above equation can be rewritten as

$$
\Delta F^{ \pm}=\overline{M T} D^{ \pm} \overline{T^{-1}} \widetilde{M^{-1}} \Delta q=\widetilde{A^{ \pm}} \Delta q
$$

Equation (7) satisfies the property 'U' of Roe (1981) and thus the flux vectors are conserved. Although the formulation becomes complicated due to transformation matrices $\bar{M}, \bar{T}$, and $\widetilde{M^{-1}}$ the differencing scheme has the representation of the convective flow propagation through these matrices and naturally allows easy characteristic boundary conditions via modified $T^{-1}$.

The implicit finite difference equation can be discretized using one side differencing depending on the sign of eigenvalues of the Jacobian matrices. Using approximate factorization, the equations are solved along the $\xi$-direction and then the $\eta$ direction successively.

The H-type grid was constructed by using elliptic P. D. E. grid generation techniques. The stretching function was used to cluster grids near the wall. The grid spacing from the wall to the closest grid is at about $y^{+}=0.5$ around the cavity. The grid system was $130 \times 70$ in the longitudinal and the normal directions of the flowfield, respectively. The incoming flow properties were prescribed using the freestream values. At the outflow boundary, the back pressure was prescribed and no-slip and adiabatic wall boundary conditions were used on the wall. In the two-equation model, turbulent kinetic energy was set to zero on the wall and wall dissipation rate was given by an asymptotic solution (Abe, Kondoh, and Nagano, 1994).

\section{RESULTS AND DISCUSSION}

\section{Characteristics of a Cascade Flow}

The general characteristics of baseline cascade flow over ARL-SL19 were analyzed by comparing with Tweedt, Schreiber, and Starken's (1988) experimental results at $M=1.59$ and $p_{2} / p_{1}=2.21$ with Baldwin-Lomax algebraic model and Abe, Kondoh, and Nagano's (1994) two-equation turbulence model. The isentropic Mach number distribution from current numerical procedure agreed quite well with experimental results as shown in Figure 1. Both turbulence models showed that the isentropic Mach number behind the $\lambda$-shock decreased rapidly at about $x / c=0.8$ on suction surface. The shock reflection on pressure surface induced pressure increase at about $x / c=0.5$. The Baldwin-Lomax model predicted nearly the normal shock structure due to thin turbulent boundary-layer thickness. The overall shock shape showed $\lambda$-shock system on the suction surface and Mach reflection (Y-shock) on the pressure surface (Figure 2). The $\lambda$-shock on suction surface changed flow pattern in the latter part of the cascade channel to trailing edge. A turbulent flow separation was induced at downstream of shock

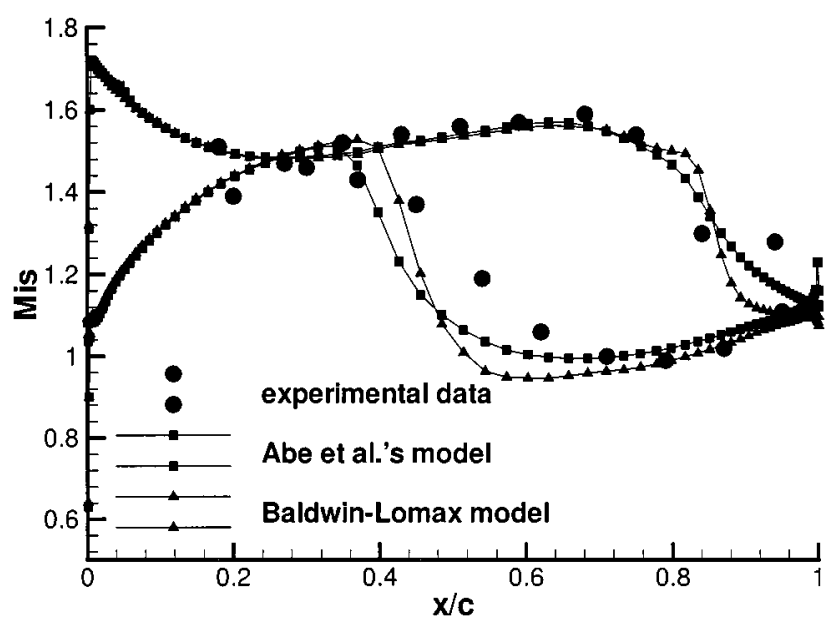

FIGURE 1

Surface isentropic Mach number comparison among experimental data and current numerical results at $M=1.59$ and $p_{2} / p_{1}=2.21$.

waves from the results of Baldwin-Lomax turbulence model. However, Abe, Kondoh, and Nagano's model did not predict flow separation though the flowfield was suddenly changed due to the $\lambda$-shock in the shock-wave/turbulent boundary-layer interaction zone. There is a tendency that eddy viscosity increases too rapidly in the interaction zone. This is why this model has some discrepancies in the prediction of the interaction between shock wave and turbulent boundary layer near flow separation zone. At increased pressure ratio $\left(p_{2} / p_{1}=2.3\right)$ Figure 3 shows the comparison of the isentropic Mach number distribution between experiment (Tweedt, Schreiber, and Starken, 1988) and prediction using Abe, Kondoh, and Nagano's turbulence model. The current model showed good agreements with experimental data.

Figure 4 shows the mass flow rate weighted total pressure losses at each grid point,

$$
\omega^{*}=\omega \times \frac{\text { local mass flow rate }}{\text { total mass flow rate }}
$$

in tangential direction in cases of the pressure ratio of 2.21 and 2.3. The peak total pressure losses were observed near the suction surface due to viscous losses from the distortion of flowfield. When the pressure ratio increased, the total pressure losses increased near suction surface due to strong flow separation but decreased at about $t=3.5 \mathrm{~cm}(t$ : distance from suction surface in tangential direction at 0.3 chord length downstream of trailing edge). The increasing exit-inlet pressure ratio caused some reduction in shock loss but slight increase in viscous loss. It is encouraging that the tendency in total pressure loss is similar to experimental results of Tweedt, Schreiber, and Starken. The total pressure losses were $\omega=0.151(=0.150$, Tweedt, Schreiber, and Starken $)$ at $p_{2} / p_{1}=2.21$ and $\omega=0.137(=0.134$, Tweedt, Schreiber, and Starken) at $p_{2} / p_{1}=2.3$. 


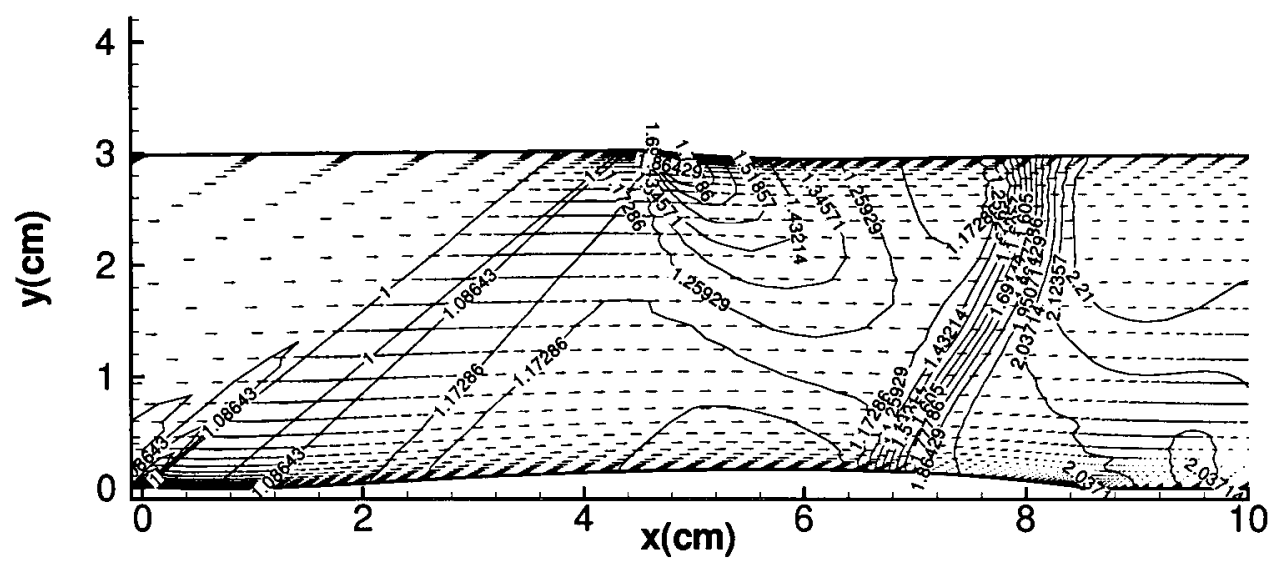

(a)

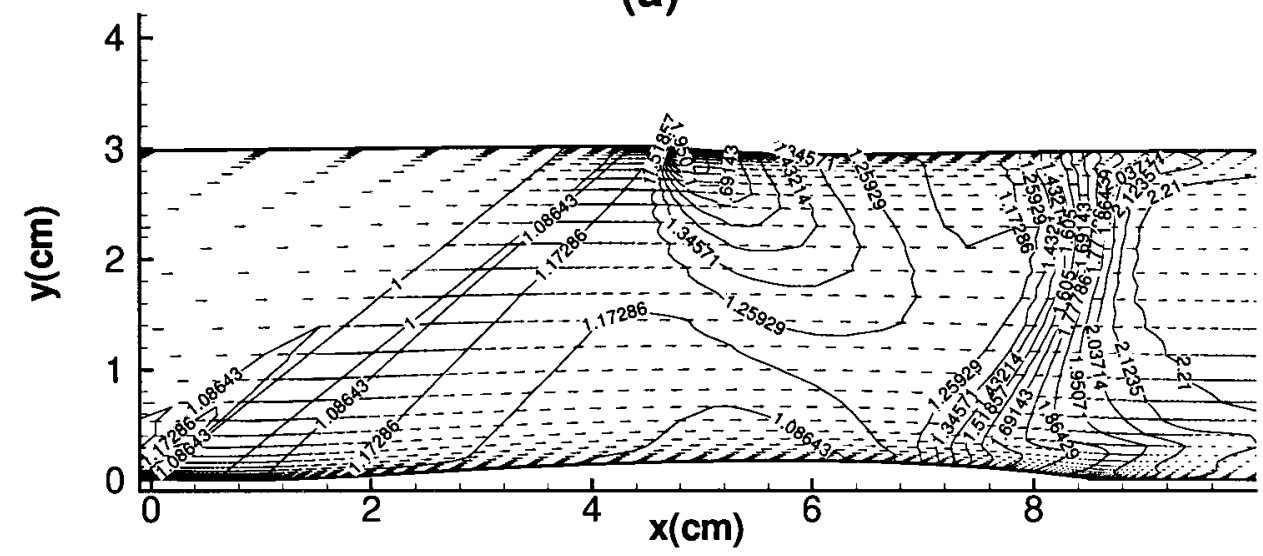

(b)

FIGURE 2

Pressure contour and velocity vector plot of (a) Baldwin-Lomax model and (b) Abe, Kondoh, and Nagano's model.

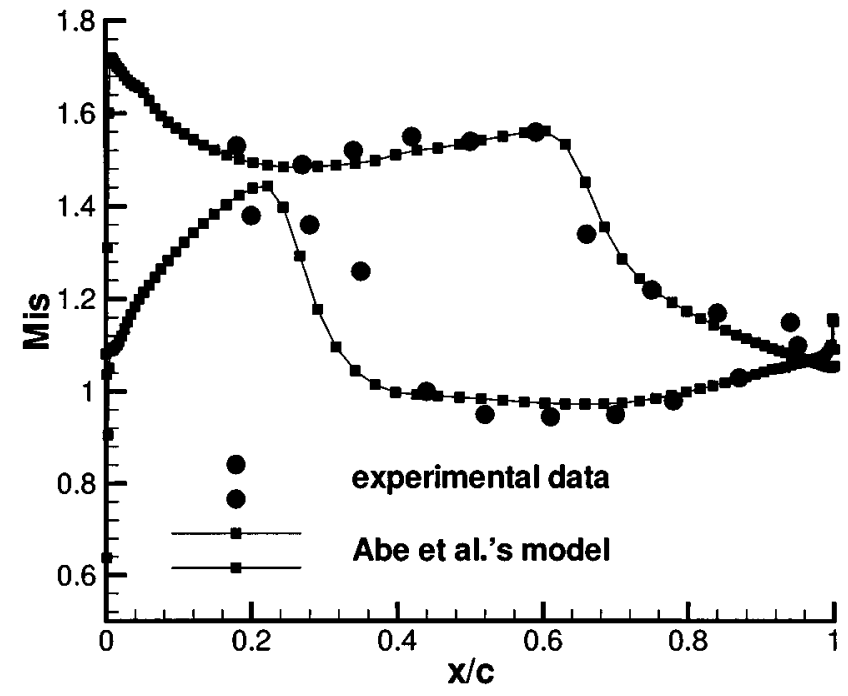

FIGURE 3

Surface isentropic Mach number comparison between experimental data and current numerical result at $M=1.59$ and $p_{2} / p_{1}=2.3$.

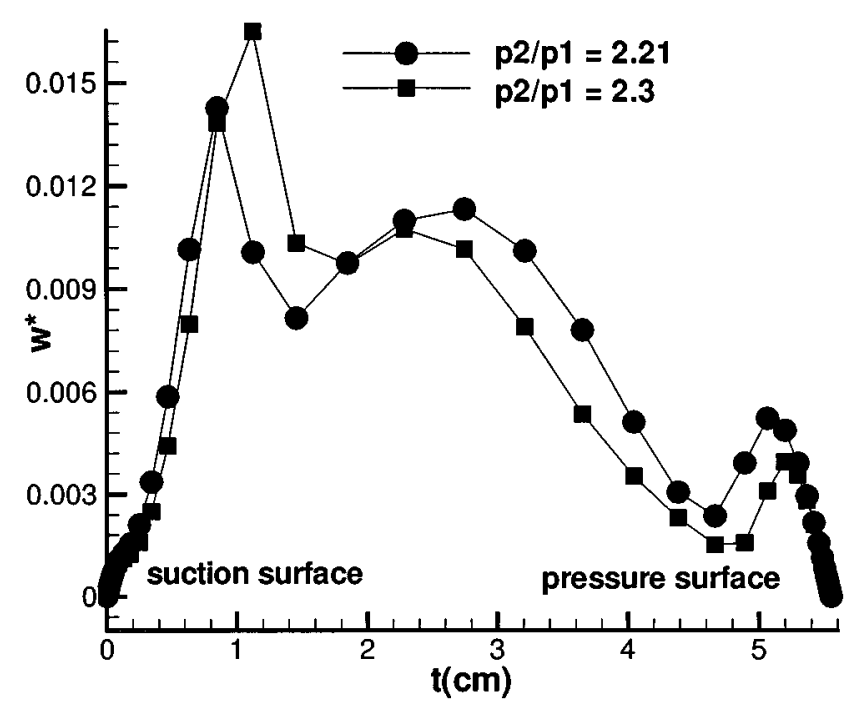

FIGURE 4

Total pressure loss coefficient comparison between different pressure ratios at $M=1.59$. 


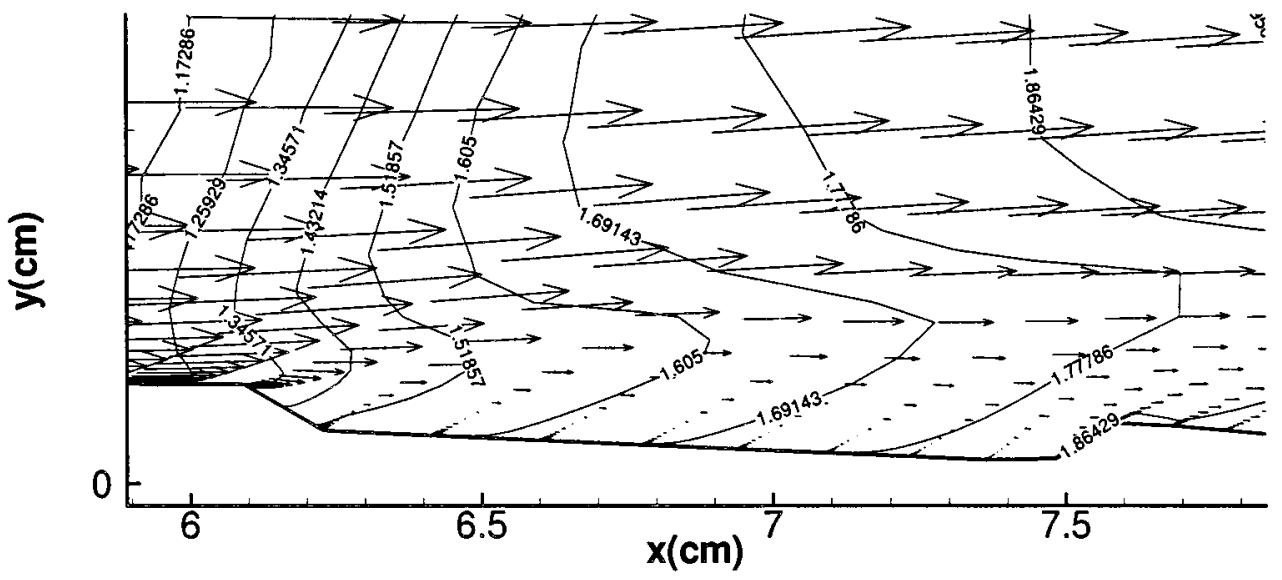

FIGURE 5

Pressure contour and velocity plot near the cavity.

\section{Effect of Cavity}

The effect of the passive control by various cavities on reducing total pressure loss is studied by varying length and depth of the cavity. The cavity controlling shock wave interaction with turbulent boundary layer reduced total pressure loss by causing more isentropic compression over cavity region. The pressure rise across the shock wave induces a passive suction downstream of the shock wave and injection of flow upstream of the shock wave through the cavity (Figure 5). The injection upstream of the shock wave over the cavity enhances boundary-layer thickening, causing a series of compression waves. This results in a shock structure that looks much like a $\lambda$-shock structure of the shock-induced separation.

The interaction length appears to somewhat increase as long as the length of each cavity. This behavior is consistent with the idea that the passive cavity spreads pressure rise over a larger axial length.

\section{Effect of Cavity Length}

Figure 6 shows the isentropic Mach number distribution comparison among the cases with different lengths of the cavity $(l / c=0.11,0.16$, and 0.2 with $d / l=0.05)$ and without cavity at $M=1.59$ and $p_{2} / p_{1}=2.21$. All the cases showed the same results before the cavity on suction surface. The weakened shock wave moved forward in front of the cavity due to the cavity effect. After the shock wave nearly the same isentropic Mach number distributions were observed up to the trailing edge. Due to weak injection of flow upstream and passive suction downstream of the cavity, the pressure contour plot showed successive compression waves over the cavity. The reflected shock waves were shown on the pressure surface due to emanating shock waves from the leading edge of the cavity. Also the reflected shock waves moved forward. Behind the shock waves the isentropic Mach number distribution to the trailing edge was almost constant on the pressure surface. Since the boundary layer became thick due to an injection and suction mechanism through the cavity, flow direction changed from solid wall and the pressure varied over wider area with cavity than without cavity in the rear part of the cascade. As the cavity length increased, the boundary layer was thickened and the pressure increased gradually over the cavity (Figures 7(a), 7(b), and 7(c)).

Figure 8 shows the comparison of the mass flow rate weighted total pressure losses at each grid point among the cases with various sized cavities and without a cavity. The peaks were shown near the suction surface in all cases. For two cases of $l / c=0.16$ and 0.2 , peak values were higher than those of no cavity and $l / c=0.11$ due to distortion of flowfield after broader shock wave/turbulent boundary-layer interaction zones. But the

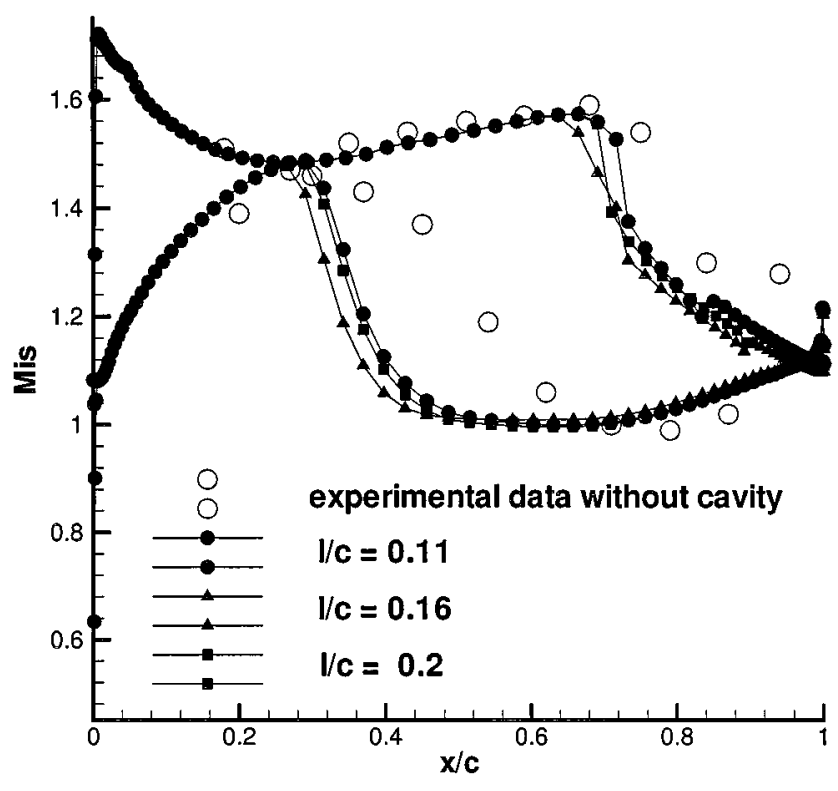

FIGURE 6

Surface isentropic Mach number comparison among the cases without and with various size cavities at $M=1.59$ and $p_{2} / p_{1}=2.21$. 


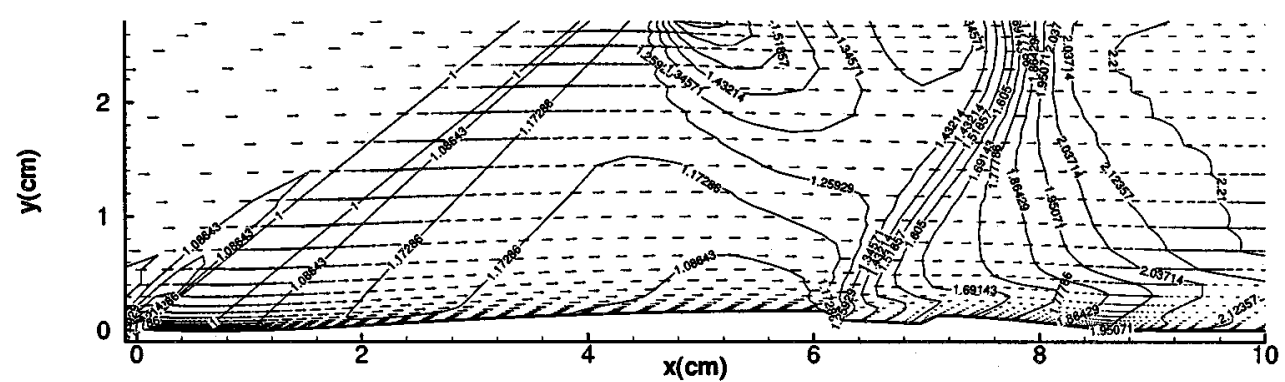

(a)

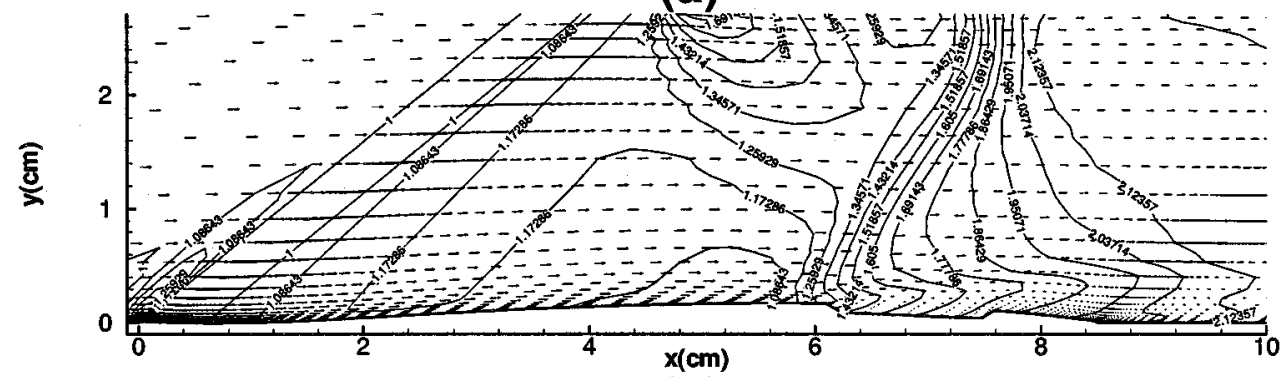

(b)

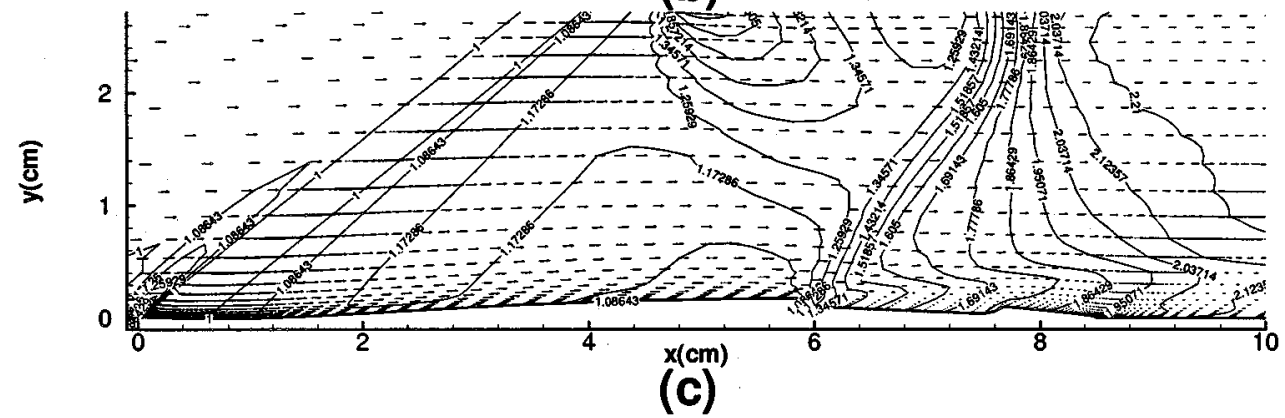

FIGURE 7

Pressure contour and velocity vector plot at the cases with (a) $l / c=0.11$, (b) $l / c=0.16$, and (c) $l / c=0.2$.

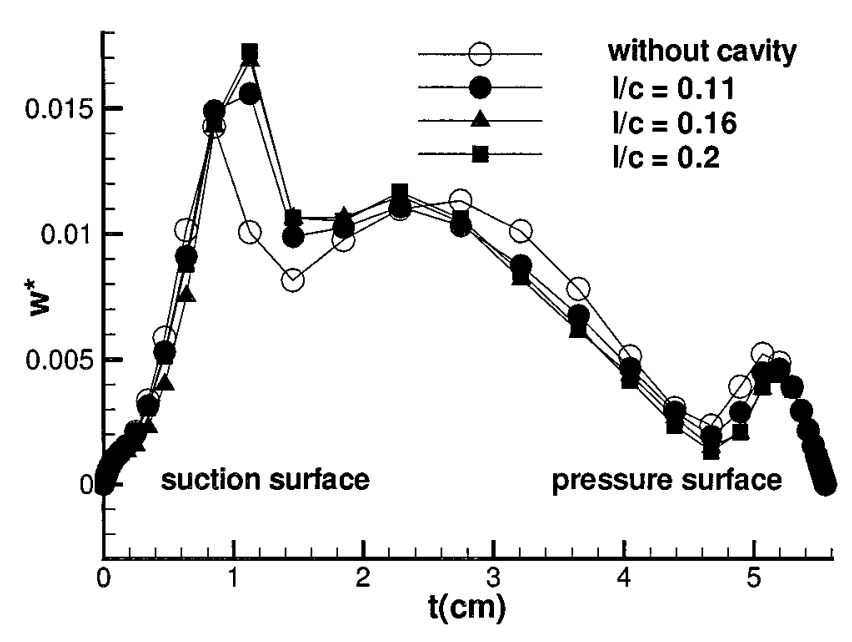

FIGURE 8

Total pressure loss coefficient comparison among the cases without and with various size cavities at $M=1.59$ and

$$
p_{2} / p_{1}=2.21 \text {. }
$$

total pressure losses decreased due to gradual increase in the pressure near the place where nearly normal shock waves were at about $t=3.5 \mathrm{~cm}$. The total pressure losses were $\omega=0.151$, 0.145 , and 0.149 at $l / c=0.11,0.16$ and 0.2 , respectively. Consequently, the cavity on the cascade reduced shock losses by inducing gradual pressure increase behind the shock wave; however, an oversized cavity could increase viscous loss due to a broader zone of shock wave/turbulent boundary-layer interaction, thus resulted in no reduction of the total pressure loss.

\section{Effect of Cavity Depth}

In case of $l / c=0.16$, the effect of cavity depth was studied by comparing $d / l=0.05$ and 0.08 cases at $M=1.59$ and $p_{2} / p_{1}=2.21$. As shown in Figure 9, the isentropic Mach number of the deep case decreased due to a shock wave further downstream than the shallow case did. Because the weak injection of flow did not thicken the boundary layer sufficiently 


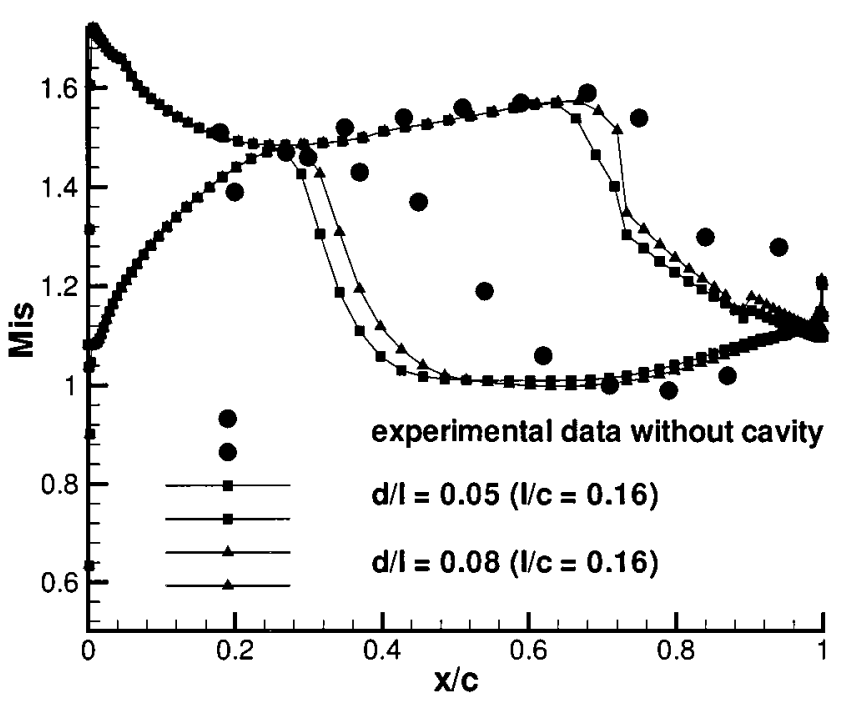

FIGURE 9

Surface isentropic Mach number comparison among the cases without and with different depth cavities at $M=1.59$ and

$$
p_{2} / p_{1}=2.21 \text {. }
$$

around the front part of the cavity and the flow expanded at the backward-facing step, the shock wave occurred downstream of the leading edge of the cavity.

Figure 10 shows the comparison of total pressure losses among the cases with different depth of the cavity and without cavity. The total pressure loss of the deep case was higher than that of the shallow case due to narrow area for the increase in the pressure. The total pressure loss for $d / l=0.08$ case was 0.152 when compared with 0.145 at $d / l=0.05$ case.

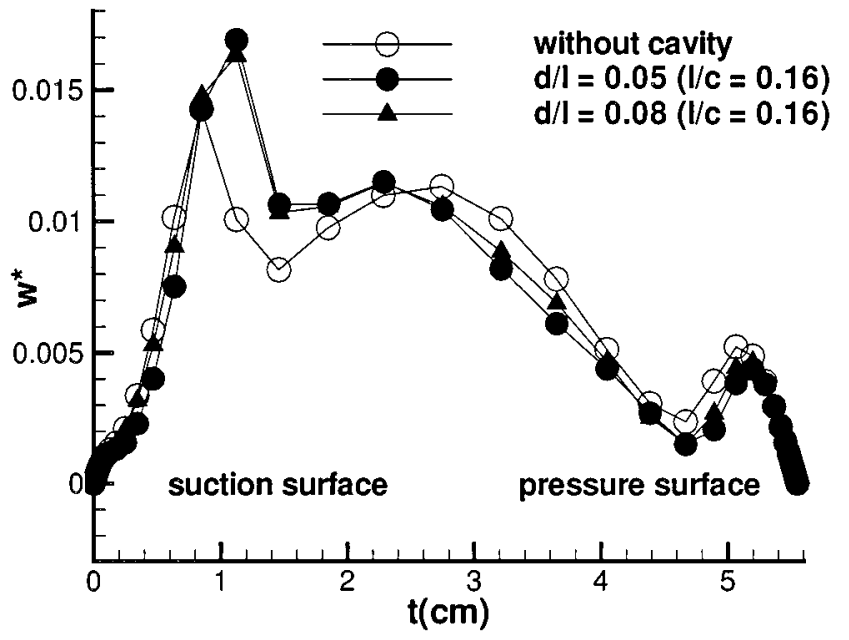

FIGURE 10

Total pressure loss coefficient comparison among the cases without and with different depth cavities at $M=1.59$ and

$$
p_{2} / p_{1}=2.21 \text {. }
$$

\section{CONCLUSIONS}

A characteristic upwind flux difference splitting NavierStokes method has been used to analyze the supersonic compressor cascade flow. The current prediction with Abe, Kondoh, and Nagano's two-equation turbulence model showed good agreement with experimental results at pressure ratios of $p_{2} / p_{1}=2.21$ and 2.3. The increasing pressure ratio caused some reduction in shock loss but slight increase in viscous loss, which is the general behavior in total pressure loss. A passive cavity was used to control the shock wave interaction with turbulent boundary layer. A $\lambda$ foot shock structure was clearly induced by a passive cavity resulting in more isentropic compression. The passive cavity reduced total pressure loss. Cavities of certain sizes could reduce total pressure losses; an oversized cavity could increase total pressure loss due to an increase in viscous loss from distortion of the cascade flowfield. The total pressure losses for $l / c=0.11,0.16$, and 0.2 were $\omega=0.151,0.145$, and 0.149 , respectively. In the effect of cavity depth $(d / l=0.05,0.08)$, the total pressure loss of the deep case was higher than that of the shallow case.

\section{NOMENCLATURE}

A Jacobian matrix

$c \quad$ sonic speed or cord length

$c_{1}, c_{2} \quad$ turbulence model constants

d cavity depth

$f_{1}, f_{2} \quad$ turbulence model functions

$F, G \quad$ inviscid fluxes

$F_{v}, G_{v}$ viscous fluxes

$k \quad$ kinetic energy

$l \quad$ cavity length

$M \quad$ Mach number

$M, T \quad$ similarity transformation matrix

$p \quad$ static pressure

$p_{t} \quad$ total pressure

$P_{k} \quad$ turbulent kinetic energy production

$q \quad$ conservative variables

$\tilde{q} \quad$ primitive variables

$\tilde{\tilde{q}} \quad$ characteristic variables

$u_{\tau} \quad$ friction velocity

$\omega \quad$ total pressure loss

$\omega^{*} \quad$ mass flow rate weighted total pressure loss

$\varepsilon \quad$ dissipation rate

$\varepsilon_{t} \quad$ volumetric total energy

$\rho \quad$ density

$\sigma_{k} \quad$ diffusion Prandtl number for turbulence energy

$\sigma_{\varepsilon} \quad$ diffusion Prandtl number for dissipation rate

$\tau \quad$ viscous stress

$\mu \quad$ viscosity

$\mu_{t} \quad$ turbulent viscosity

$\xi, \eta \quad$ axis in general curvilinear coordinate system

$\Delta \quad$ spatial difference 


\section{REFERENCES}

Abe, K., Kondoh, T., and Nagano, Y. 1994. A new turbulence model for predicting fluid flow and heat transfer in separating and reattaching flows-I., Flow field calculations. International Journal Heat Mass Transfer 37(1):139-151.

Bahi, L., Ross, J. M., and Nagamatsu, H. T. 1983. Passive shock wave/boundary layer control for transonic airfoil drag reduction, AIAA Paper 83-0137.

Godunov, S. K. 1959. A finite difference method for the numerical computation of discontinuous solutions of the equations of fluid dynamics. Mat. Sb.:271-290.

Kim, S. D., Kwon, C. O., Song, D. J., and Sa, J. Y. 1995. Performance enhancement study using passive control of shock-boundary layer interaction in a transonic/supersonic compressor cascade. Trans. KSME 20:2944-2952.

Kim, S. D., and Song, D. J. 1997. Comparative study on two-equation turbulence models for transonic/supersonic flows. Proceeding of the KSCFE Spring Annual Meeting:25-29.

Lombard, C. K., Bardina, J., Venkatapathy, E., and Oliger, J. 1983. Multi-dimensional formulation of CSCM-An upwind flux difference eigenvector split method for the compressible navier-stokes equations. AIAA-83-1859cp.

McComick, D. C. 1993. Shock/Boundary-Layer Interaction control with Vortex Generators and Passive Cavity. AIAA Journal 31:9196.
Nagamatsu, H. T., Orozco, R. D., and Ling, D. C. 1984. Porosity effect on supercritical airfoil drag reduction by shock wave/boundary layer control. AIAA Paper 84-1682.

Osher, S. 1984. Riemann solvers, the entropy condition, and difference approximations. SIAM Journal of Numerical Analysis 21:217235.

Raghunathan, S. 1989. Passive control of shock-boundary layer interaction. Progress in Aerospace Sciences 25:271-296.

Roe, P. L. 1981. The use of the Riemann problem in finite-difference schemes. Proceedings of the 7th International Conference on Numerical Methods in Fluid Dynamics, Lecture Notes in Physics, 141:354-359.

Schnerr, G. H., and Dohrmann, D. 1993. Numerical and experimental investigation of passive control of the shock-boundary layer interaction in a transonic compressor cascade. Proceedings of 2nd ICFM: 504-510.

Steger, J. L., and Warming, R. F. 1981. Flux vector splitting of the inviscid gasdynamics equations with application to finite difference methods. Journal Computational Physics 40:263293.

Tweedt, D. L., Schreiber, H. A., and Starken, H. 1988. Experimental investigation of the performance of a supersonic compressor cascade. Journal of Turbomachinery, Trans. ASME 110:456-466.

Van Leer, B. 1982. Flux-vector splitting for Euler equations. Lecture Notes in Physics 170:507-512. 

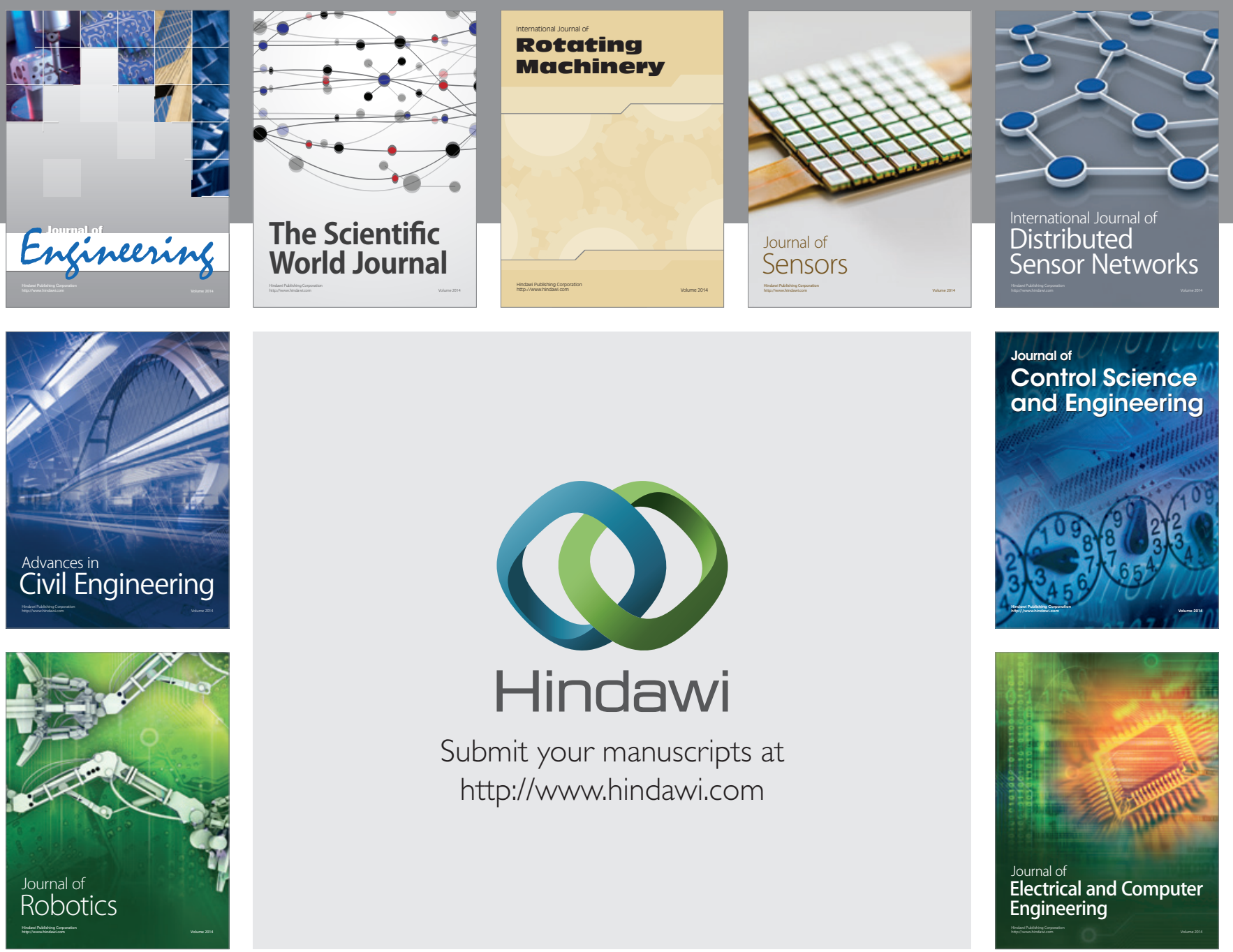

Submit your manuscripts at

http://www.hindawi.com
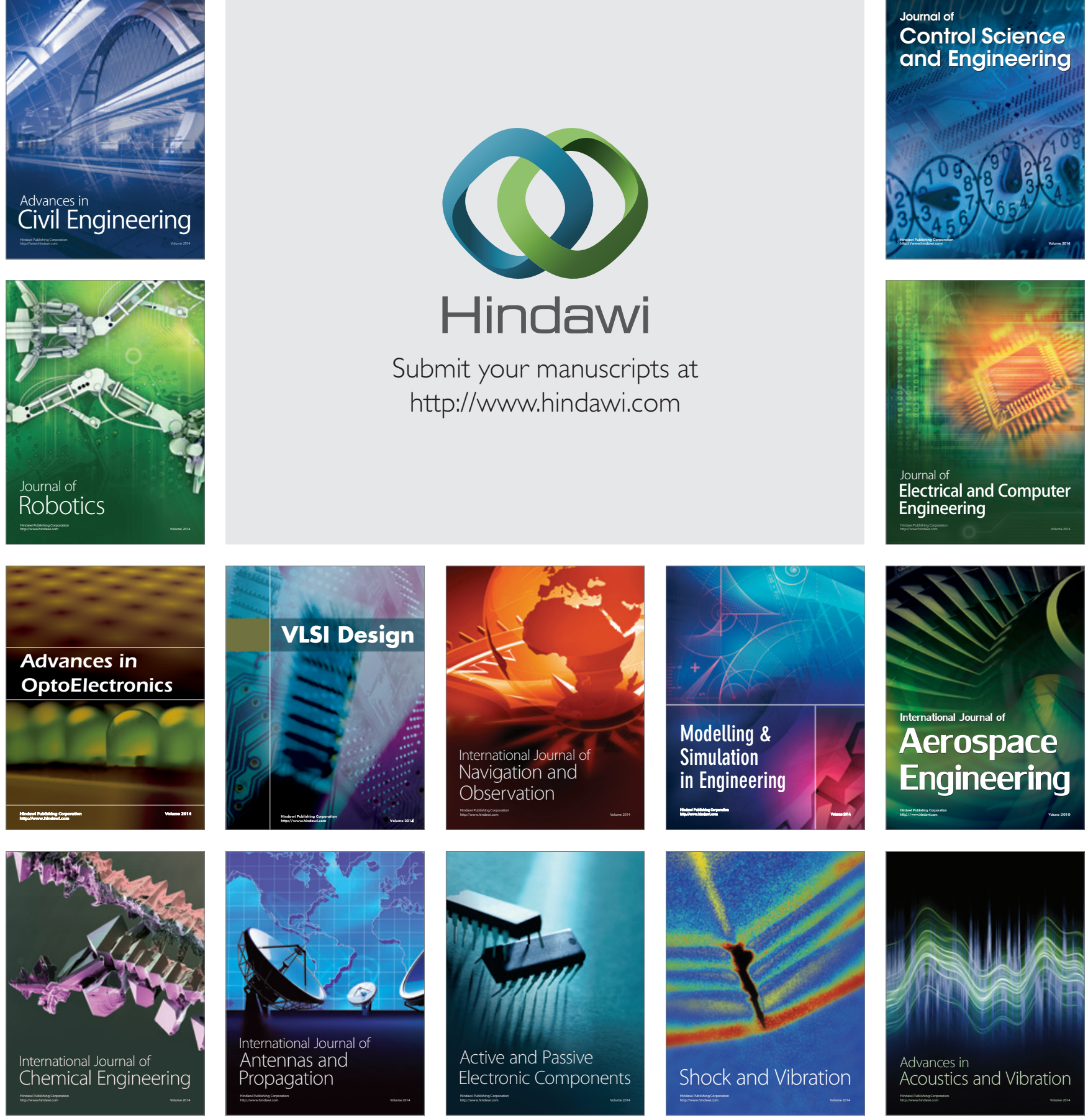